\title{
Twiddler's Syndrome in a Patient with Dystonic Tremor Treated with DBS
}

\author{
Jennifer Samuelsson ${ }^{*}$, Patric Blomstedt ${ }^{2}$ \\ ${ }^{1}$ Department of Neurosurgery, Umeå University Hospital, Umeå, Sweden \\ ${ }^{2}$ Department of Neurosurgery, Umeå University, Umeå, Sweden \\ Email: “Jennifer.samuelsson@neuro.umu.se, patric.blomstedt@neuro.umu.se
}

Received 5 August 2014; revised 5 September 2014; accepted 5 October 2014

Copyright (C) 2014 by authors and Scientific Research Publishing Inc.

This work is licensed under the Creative Commons Attribution International License (CC BY). http://creativecommons.org/licenses/by/4.0/

(c) (i) Open Access

\begin{abstract}
Background and Importance: Twiddler's syndrome is a rare complication of DBS. This condition occurs when the IPG is consciously or inadvertently rotated in its pocket, resulting in torsion and possible dislodgement of implanted electrodes, with subsequent loss of function. Methods: Here we present a patient diagnosed with Twiddler's syndrome. The patient presented with straining cables at the neck five months after bilateral Gpi DBS and an x-ray demonstrated Twiddler's syndrome. Initial revision with preventive measures proved futile. After some time the condition recurred, now with dislocation of one of the intracerebral electrodes. In a second revision the IPG was placed under the pectoralis muscle, which has so far prevented further rotation. Results and Conclusion: While Twiddler's syndrome is fairly uncommon, it remains to be a risk associated with DBS, recognizing the potential risks and signs might allow for preventive measures avoiding dislocation of the intracerebral electrodes.
\end{abstract}

\section{Keywords}

Twiddler's Syndrome, IPG, DBS, Complications

\section{Introduction}

Twiddler's syndrome, first time described in 1968 [1], is a rare complication of DBS. This condition occurs when the IPG is consciously or inadvertently rotated in its pocket, resulting in torsion and possible dislodgement of implanted electrodes, with subsequent loss of function.

In this case report we present a patient diagnosed with Twiddler's syndrome five months after the implantation and where the initial revision proved futile, leading to eventual dislocation of the intracerebral electrode.

${ }^{*}$ Corresponding author.

How to cite this paper: Samuelsson, J. and Blomstedt, P. (2014) Twiddler's Syndrome in a Patient with Dystonic Tremor Treated with DBS. Open Journal of Modern Neurosurgery, 4, 193-195. http://dx.doi.org/10.4236/ojmn.2014.44034 


\section{Case Report}

A patient with dystonic tremor mainly in the head and upper extremities was implanted with bilateral electrodes (model 3389, Medtronic). An intra-operative CT fused with the pre-operative MR demonstrated the electrodes to be located in the intended target.

The electrodes were connected with two elastic extension cables (Model 37086, Medtronic) to a PC IPG (Medtronic). The IPG was placed in a subcutaneous pocket below the left clavicle on the fascia of the pectoralis muscle. The patient had a rather thick layer of subcutaneous fat and the area was very mobile, due to a large bosom.

Approximately 5 months after the procedure the patient experienced rotation of the pacemaker, sometimes necessitating manual correction on the patients behalf in order to get it back into the proper position. The patient did further experience tenderness behind the left ear above the connection between the extension cables and electrodes. An x-ray demonstrated rotation of the IPG with multiple twisting of the extension cables, however without affection of the intracerebral electrodes.

During the revision, the left extension cable was replaced and the IPG was put further down behind the breast, attached with two non-absorbable sutures and the IPG pocket was tightened.

Approximately seven months later the patient once again suffered the same symptoms. Imaging revealed an even more pronounced twisting of the extension cables and IPG (Figure 1(C)).The right intracerebral electrode was no longer in its original place, but had been pulled out 3 - 4 centimetres (Figure 1(A)). No apparent change was seen regarding the effect of DBS. The head tremor was still abolished, and no clear effect had ever been seen regarding the minor tremor in the right upper extremity.

During the second revision, the IPG was found rotated with the non-absorbable sutures ruptured (Figure 1(B)). The cable was re-winded and the IPG was this time implanted beneath the pectoralis muscle, fixated with non-absorbable sutures. An X-ray performed after three months demonstrated no signs of rotation of the IPG in its current position and no further clinical symptoms have emerged during the follow-up of 6 months.

\section{Discussion}

Twiddler's syndrome is a rare complication in DBS. It is caused by the rotation (intentionally or unintentionally) of the IPG around its long axis, sometimes causing dislocation of the electrodes.

However, this complication is well-known regarding cardiac pacemakers, where it is reported to occur in $0.7 \%$ - 7\% of the patients.

Most patients are diagnosed within the first year and are more likely to develop in women who tend to have loose subcutaneous tissue [2]. Other risk factors facilitating rotation of the IPG include obesity, excessive movements of the upper limbs, increasing age, large size pockets and mentally unstable patients actively manipulating the IPG [3] [4]. Most of these risk factors cannot be avoided and in high-risk patients preventive measures might be considered, such as minimizing the IPG-pocket, attaching the IPG to the fascia or placing it sub-pectorally [3]. However, not even a placement below the muscle has proven to be a guarantee in the experience of cardiac pacemakers [3].

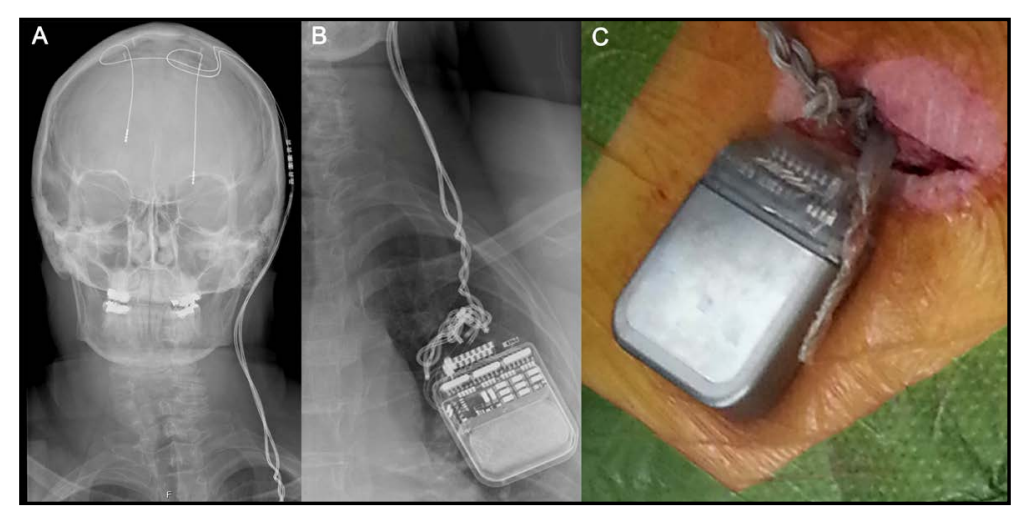

Figure 1. Demonstrating the dislocation of the left DBS-electrode (A), and the rotation of the extension cables on a plain X-ray (B) and during surgery (C). 
This condition can easily be diagnosed radio-graphically and will often present itself clinically in a most distinct manner, with straining cables in the neck and a palpable coil of cables at the IPG. It should also be considered as a possibility in cases of sudden deterioration of the effect of DBS, or new stimulation induced side effects.

\section{Conclusion}

While Twiddler's syndrome is fairly uncommon, it remains to be a risk associated with DBS. Recognizing the potential risks and signs might allow for preventive measures avoiding dislocation of the intracerebral electrodes.

\section{References}

[1] Fahraeus, T. and Hijer, C.J. (2003) Early Pacemaker Twiddler Syndrome. Europace, 5, 279-281. http://dx.doi.org/10.1016/S1099-5129(03)00032-1

[2] Astradsson, A., Schweder, P.M., Joint, C., et al. (2011) Twiddler's Syndrome in a Patient with a Deep Brain Stimulation Device for Generalized Dystonia. Journal of Clinical Neuroscience, 18, 970-972. http://dx.doi.org/10.1016/j.jocn.2010.11.012

[3] Geissinger, G. and Neal, J.H. (2007) Spontaneous Twiddler's Syndrome in a Patient with a Deep Brain Stimulator. Surgical Neurology, 68, 454-456. http://dx.doi.org/10.1016/j.surneu.2006.10.062

[4] Gelabert-Gonzalez, M., Relova-Quinteiro, J.L. and Castro-Garcia, A. (2010) “Twiddler's Syndrome” in Two Patients with Deep Brain Stimulation. Acta Neurochirurgica (Wien), 152, 489-491.

http://dx.doi.org/10.1007/s00701-009-0366-6 
Scientific Research Publishing (SCIRP) is one of the largest Open Access journal publishers. It is currently publishing more than 200 open access, online, peer-reviewed journals covering a wide range of academic disciplines. SCIRP serves the worldwide academic communities and contributes to the progress and application of science with its publication.

Other selected journals from SCIRP are listed as below. Submit your manuscript to us via either submit@scirp.org or Online Submission Portal.
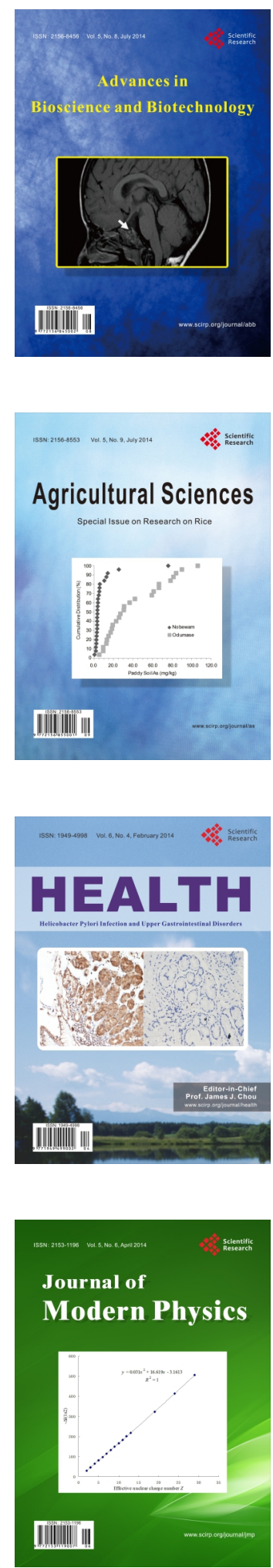
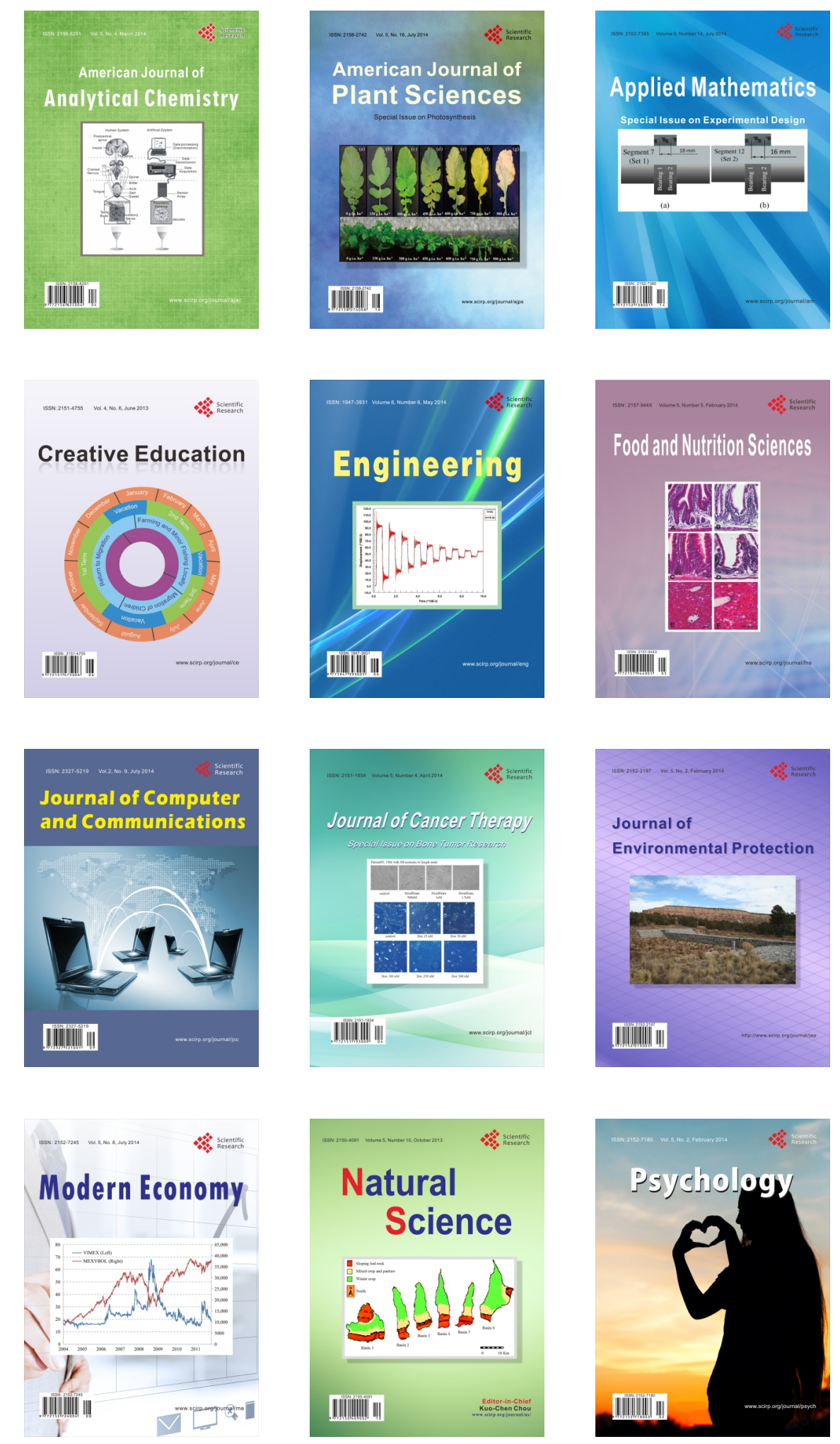